\title{
Travelling Waves in Plankton Dynamics
}

\author{
M. Semplice, E. Venturino * \\ Dipartimento di Matematica "Giuseppe Peano" \\ Università di Torino, Italy
}

\begin{abstract}
A recently proposed model for the investigation of diffusivity in planktonic systems containing toxin-producing phytoplanktons is here reconsidered. We show the existence of planktonic travelling waves. Numerical simulations validate the analytical findings, to elucidate the sensitivity of the results in dependence of the diffusion coefficients.
\end{abstract}

Keywords and phrases: competition models, food web, toxic phytoplankton, minimal models

Mathematics Subject Classification: 92D25, 92D40, 35Q92

\section{Introduction}

In the past quarter of century research on plankton dynamics has become relevant, both at the biological level, as well as from the modelling point of view. Plankton has indeed a paramount importance in the aquatic food webs, since it represents the lower trophic level in such chains. Mathematical models for planktonic systems including space dependence often lead to the discovery of insurgences of patterns, see the second half of [7] for an account of these topics, both from the deterministic and stochastic points of view.

A phenomenon that has negative impact on tourism and the fisheries industries is due to red tides, the sudden blooms of algae, $[1,6,11]$. Some species of phytoplankton can release poisons that affect zooplankton as well as possibly, directly or indirectly, also fish. Recent investigations have shown that movement can sustain inhomogeneous distribution levels of plankton communities composed of nontoxic phytoplankton, toxic phytoplankton and zooplankton, $[3,10]$. An idea that has been proposed for explaining the insurgence of red tides is that they are side effects caused by these toxin producing phytoplanktons, $[4,5,8,9]$.

In this paper we develop further the work undertaken in [2] on diffusive plankton movements, for which the action of the toxin-producing phytoplankton not only damages the grazers, but it can also cause spatial inhomogeneities. In [2] the steady states of this plankton model have been investigated and the corresponding spatiotemporal patterns discovered. Here, we study the possible insurgence of planktonic travelling waves, that ultimately produce these steady state distributions.

*Corresponding author. E-mail: matteo.semplice@unito.it, ezio.venturino@unito.it 
The results show that when a new species appears at a single location in a system that is at equilibrium, it may originate an invading wave. We confirm previous findings and exhibit traveling waves that leave behind them the non spatially-uniform coexistence equilibrium of the toxic model.

The paper has the following structure. The next Section presents the model, which is analysed in Section 3. Travelling waves are investigated in Section 4 for the nontoxic model and in Section 5 for the toxic one. Then numerical simulations follow, to substantiate and extend the analytical findings. The sensitivity of the results on the diffusion coefficients is also investigated.

\section{The population model}

We consider a model for three interacting plankton species of the form:

$$
\frac{\partial p_{k}}{\partial t}=f_{k}(\mathbf{p})+D_{k} \Delta p_{k}, \quad k=1,2,3
$$

where $\Delta$ represents the Laplacian and $\mathbf{p}(t, x)=\left(p_{1}(t, x), p_{2}(t, x), p_{3}(t, x)\right)$ are the densities of each population at location $x$ in space, $D_{k}$ are diffusion coefficients and the reaction terms described by $f_{k}$ model the interactions among the populations. Since (2.1) represents a population model, the densities must be nonnegative, $p_{i}(t, x) \geq 0, i=1,2,3$ for $(t, x) \in\left\{\mathbf{R}^{+} \times \mathbf{R}^{+}\right\}$, as well as the initial conditions, $p_{i}(0, x)=p_{i}^{(0)}(x)$ where the latter represent given functions.

More precisely we consider the model studied in [2] where $p_{1}$ and $p_{2}$ are phytoplankton species and $p_{3}$ reprents zooplankton that feeds on the other two. In [2] two versions of the model are considered: the case where $p_{2}$ is toxic for the zooplankton is compared to the standard situation.

$$
\begin{aligned}
f_{1}^{(\mathrm{std})}(\mathbf{p})=f_{1}^{(\mathrm{tox})}(\mathbf{p}) & =p_{1}\left[r_{1}\left(1-\frac{p_{1}+\psi_{1} p_{2}}{K}\right)-\alpha_{1} p_{3}\right] \\
f_{2}^{(\mathrm{std})}(\mathbf{p})=f_{2}^{(\mathrm{tox})}(\mathbf{p}) & =p_{2}\left[r_{2}\left(1-\frac{p_{2}+\psi_{2} p_{1}}{K}\right)-\alpha_{2} p_{3}\right] \\
f_{3}^{(\text {std })}(\mathbf{p}) & =p_{3}\left(\beta p_{1}-c+\delta p_{2}\right) \\
f_{3}^{(\text {tox })}(\mathbf{p}) & =p_{3}\left(\beta p_{1}-c-\frac{\theta p_{2}^{2}}{m+p_{2}}\right)
\end{aligned}
$$

In these models it is assumed that both harmless and toxic phytoplankton live in the same environment and share common resources, for which the first two equations in (2.2) exhibit the same carrying capacity $K$. They do reproduce at different rates, however. Respectively, the latter are $r_{1}$ and $r_{2}$. The coefficient $\psi_{1}$ measures in a sense the competition ability of the phytoplankton $p_{2}$ over the phytoplankton population $p_{1}$, or in other words the damage that the former inflicts to the latter. A similar description holds conversely for $\psi_{2}$. The parameters $\alpha_{i}, i=1,2$ represent the grazing rates of zooplankton over each phytoplankton population. The $p_{1}$ biomass is then converted into new zooplankton via the uptake rate $\beta$. The natural zooplankton mortality is denoted by $c$. The last terms in the last two equations of (2.2) differ in sign, as they model two different situations. The former describes the standard situation, $p_{2}$ is converted into new zooplankton biomass at rate $\delta$. The latter instead states that toxic phytoplankton kills the zooplankton at rate $\theta$. In this case, also the functional form is different. In [2] the linear form has been chosen for the standard case, while the multiplier

$$
\frac{p_{2}}{m+p_{2}}
$$

has the form of a Holling type II response function, where $m$ represents the half-saturation constant. Thus it tends to vanish for a small toxic phytoplankton population, while it exhibits a saturation phenomenon when the population becomes very large. Hence, the multiplier is not constant, but an implicit nonlinear function of time. The reason might be due to the fact that for the poison to be effectively released, a small number of toxic phytoplankton is not sufficient. At the same time, when the harmful phytoplanktons are 

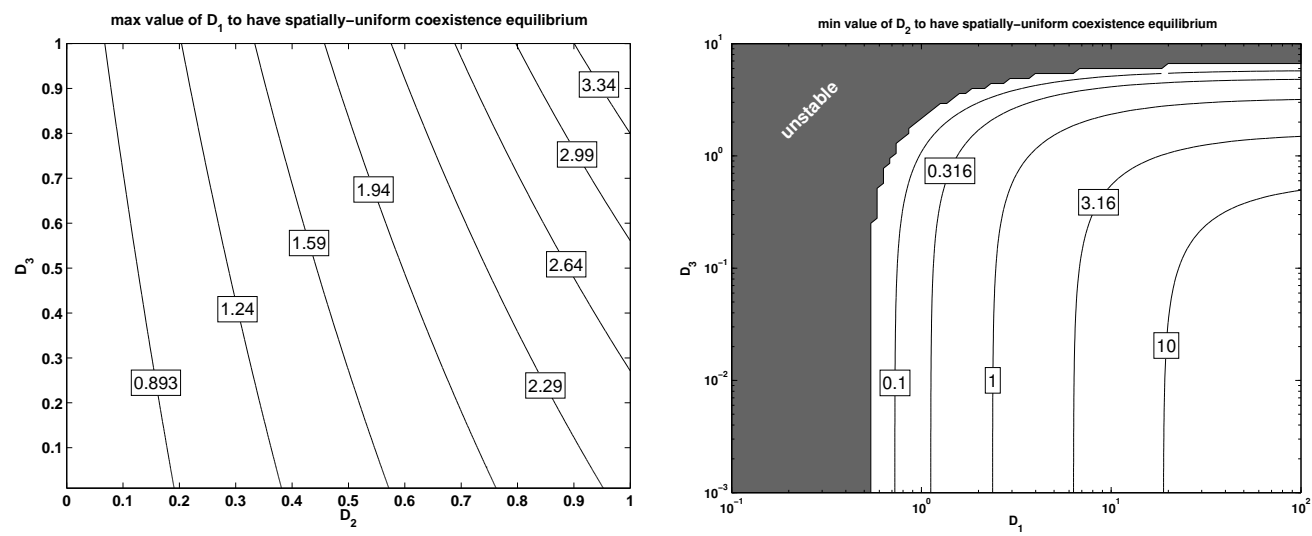

Figure 1. Threshold values of the diffusion coefficients for the stability of the spatially stable uniform coexistence equilibrium in the toxic model. Left: threshold for $D_{1}$ as a function $D_{2}$ and $D_{3}$. Right: threshold for $D_{2}$ as a function $D_{1}$ and $D_{3}$. In the shaded region on the right, the stable coexistence equilibrium in always non-uniform.

in large numbers, the assumption in (2.2) is that there is a maximal toxin release rate. Other options could have been simply again a linear rate or, alternatively, a Holling type II response function, for which the square in the numerator would not appear in the last equation of (2.2). Taking these models from the available literature, in this paper we investigate how the spatial configurations of these systems evolve in time, disregarding the fact that they do not contain the same functional response.

In [2] it is shown that both models admit a spatially uniform stable state corresponding to a nontrivial coexistence equilibrium of the three species, but that in the scenario where the second phytoplankton species is toxic, this is stable only if the mobility $D_{1}$ of the nontoxic prey is smaller than a threshold value that depends on the parameters in the reaction terms and on the other two diffusion coefficients. Figure 1 depicts such threshold as a function of $D_{2}$ and $D_{3}$ for the values of the parameters used in [2] and in the numerical tests of this paper (see Sec. 6)

\section{Travelling waves}

In this paper we concentrate on the regimes where a spatially uniform coexistence equilibrium is spatially stable, i.e. we restrict to $D_{1}$ small enough in the toxic scenario, and show that travelling wave solutions representing the invasion of the spatial domain by the $p_{2}$ species are allowed in the model.

By travelling wave solution we mean a solution of the form

$$
p_{k}(t, x)=\hat{p}_{k}(x-v t)=P_{k}(s)
$$

for some constant speed $v$ with $\hat{p}_{k}$ constant away from the origin and "linking" the initial data with the stable equilibrium:

$$
\lim _{\xi \rightarrow-\operatorname{sgn}(v) \cdot \infty} \hat{p}_{k}(\xi)=p_{k}^{\mathrm{eq}} \quad \lim _{\xi \rightarrow+\operatorname{sgn}(v) \cdot \infty} \hat{p}_{k}(\xi)=p_{k}^{\text {initial }}
$$

Ecologically, this means that the appearence of individuals of the $p_{2}$ population, for instance, in a given domain (where $p_{1}$ and $p_{3}$ are in equilibrium) gives rise to an invasion front moving at constant speed and separating the initial state (ahead of the front) and the nontrivial equilibrium (behind the front).

On differentiating (3.1) we have

$$
\frac{\partial \hat{p}_{k}}{\partial t}=-v P_{k}^{\prime}, \quad \frac{\partial^{2} \hat{p}_{k}}{\partial x^{2}}=P_{k}^{\prime \prime}
$$


We can now rewrite the first two equations in the systems in terms of the new functions $P_{k}$ as follows

$$
\begin{aligned}
& -v P_{1}^{\prime}=P_{1}\left[r_{1}-\frac{r_{1}}{K}\left(P_{1}+\psi_{1} P_{2}\right)-\alpha_{1} P_{3}\right]+D_{1} P_{1}^{\prime \prime} \\
& -v P_{2}^{\prime}=P_{2}\left[r_{2}-\frac{r_{2}}{K}\left(P_{2}+\psi_{2} P_{1}\right)-\alpha_{2} P_{3}\right]+D_{2} P_{2}^{\prime \prime}
\end{aligned}
$$

while the third equation in the non-toxic case is

$$
-v P_{3}^{\prime}=P_{3}\left[\beta P_{1}+\delta P_{2}-c\right]+D_{3} P_{3}^{\prime \prime}
$$

and in the toxic case instead is

$$
-v P_{3}^{\prime}=P_{3}\left[\beta P_{1}-\frac{\theta}{m+P_{2}} P_{2}^{2}-c\right]+D_{3} P_{3}^{\prime \prime} .
$$

Defining $Q_{k}=P_{k}^{\prime}$ for $k=1,2,3$, we obtain the systems

$$
\begin{array}{r}
P_{k}^{\prime}=Q_{k} \\
Q_{1}^{\prime}=\frac{1}{D_{1}} P_{1}\left[\alpha_{1} P_{3}+\frac{r_{1}}{K}\left(P_{1}+\psi_{1} P_{2}\right)-r_{1}\right]-\frac{v}{D_{1}} Q_{1} \\
Q_{2}^{\prime}=\frac{1}{D_{2}} P_{2}\left[\alpha_{2} P_{3}+\frac{r_{2}}{K}\left(P_{2}+\psi_{2} P_{1}\right)-r_{2}\right]-\frac{v}{D_{2}} Q_{2} \\
Q_{3}^{\prime}=\frac{1}{D_{3}} P_{3}\left[c-\beta P_{1}-\delta P_{2}\right]-\frac{v}{D_{3}} Q_{3},
\end{array}
$$

in the non-toxic case and instead for the toxic case

$$
\begin{array}{r}
P_{k}^{\prime}=Q_{k} \\
Q_{1}^{\prime}=\frac{1}{D_{1}} P_{1}\left[\alpha_{1} P_{3}+\frac{r_{1}}{K}\left(P_{1}+\psi_{1} P_{2}\right)-r_{1}\right]-\frac{v}{D_{1}} Q_{1} \\
Q_{2}^{\prime}=\frac{1}{D_{2}} P_{2}\left[\alpha_{2} P_{3}+\frac{r_{2}}{K}\left(P_{2}+\psi_{2} P_{1}\right)-r_{2}\right]-\frac{v}{D_{2}} Q_{2} \\
Q_{3}^{\prime}=\frac{1}{D_{3}} P_{3}\left[c-\beta P_{1}+\frac{\theta}{m+P_{2}} P_{2}^{2}\right]-\frac{v}{D_{3}} Q_{3} .
\end{array}
$$

\section{Waves for the non-toxic model}

We investigate the equilibria of (3.5) at first. For all of them we must have $Q_{k}=0$, and then seek the population levels $P_{k}$ that annihilate the right hand sides of the system. Restricting just to the $P_{1}-P_{2}-P_{3}$ phase subspace, we find the points $X_{0} \equiv(0,0,0), X_{1} \equiv(K, 0,0), X_{2} \equiv(0, K, 0)$, always feasible,

$$
X_{3} \equiv\left(0, \frac{c}{\delta},\left(1-\frac{c}{\delta K}\right) \frac{r_{2}}{\alpha_{2}}\right), \quad X_{4} \equiv\left(\frac{c}{\beta}, 0,\left(1-\frac{c}{\beta K}\right) \frac{r_{1}}{\alpha_{1}}\right)
$$

which are feasible respectively for

$$
\delta K>c
$$

and

$$
\beta K>c
$$

We find also

$$
X_{5} \equiv\left(\frac{K}{\Delta}\left(1-\psi_{1}\right), \frac{K}{\Delta}\left(1-\psi_{2}\right), 0\right), \quad \Delta=1-\psi_{1} \psi_{2}
$$


feasible when either one of the two following alternative sets of conditions holds

$$
1>\psi_{1}, \quad 1>\psi_{2} ; \quad 1<\psi_{1}, \quad 1<\psi_{2} .
$$

In addition there is the point $X_{6}$ with all populations at nonzero levels.

The Jacobian of (3.5) in the phase space $P_{1}-P_{2}-P_{3}-Q_{1}-Q_{2}-Q_{3}$ is a partitioned matrix in $3 \times 3$ blocks

$$
J=\left(\begin{array}{ll}
O & I \\
A & B
\end{array}\right)
$$

where

$$
A=\left(\begin{array}{ccc}
A_{11} & \frac{r_{1} \psi_{1}}{K D_{1}} P_{1} & \frac{\alpha_{1}}{D_{1}} P_{1} \\
\frac{r_{2} \psi_{2}}{K D_{2}} P_{2} & A_{22} & \frac{\alpha_{2}}{D_{2}} P_{2} \\
-\frac{\beta}{D_{3}} P_{3} & -\frac{\delta}{D_{3}} P_{3} & A_{33}
\end{array}\right)
$$

with

$$
\begin{array}{r}
A_{11}=\frac{1}{D_{1}}\left(\alpha_{1} P_{3}+r_{1} \frac{2}{K} P_{1}+\frac{r_{1} \psi_{1}}{K} P_{2}-r_{1}\right), \\
A_{22}=\frac{1}{D_{2}}\left(\alpha_{2} P_{3}+r_{2} \frac{2}{K} P_{2}+\frac{r_{2} \psi_{2}}{K} P_{1}-r_{2}\right), \\
A_{33}=\frac{1}{D_{3}}\left(c-\beta P_{1}-\delta P_{2}\right),
\end{array}
$$

and

$$
B=\operatorname{diag}\left(-\frac{v}{D_{1}},-\frac{v}{D_{2}},-\frac{v}{D_{3}}\right) .
$$

Exchanging the pairs of rows and columns 2 and 4, then 4 and 6 , followed by a block interchange of the last two rows and columns, and finally exchanging the rows and columns 3 and 4 , the matrix $J-\lambda I$ is reduced to block form, in which each block is a 2 by 2 matrix. Its final form is as follows

$$
J-\lambda I=\left(\begin{array}{cccccc}
-\lambda & 1 & 0 & 0 & 0 & 0 \\
A_{11}-\frac{v}{D_{1}}-\lambda & A_{12} & 0 & A_{13} & 0 \\
0 & 0 & -\lambda & 1 & 0 & 0 \\
A_{21} & 0 & A_{22} & -\frac{v}{D_{2}}-\lambda & A_{23} & 0 \\
0 & 0 & 0 & 0 & -\lambda & 1 \\
A_{31} & 0 & A_{32} & 0 & A_{33}-\frac{v}{D_{3}}-\lambda
\end{array}\right) .
$$

This corresponds also to a rearrangement of the vector of the unknown populations, as follows: $\left(P_{1}, P_{1}^{\prime}, P_{2}, P_{2}^{\prime}, P_{3}, P_{3}^{\prime}\right)^{T}$. Although for our purposes the case of the origin is not very much interesting, we present it nevertheless, in order to better illustrate the procedure we use in the relevant cases that follow. At the origin, only the diagonal blocks in the Jacobian are nonzero,

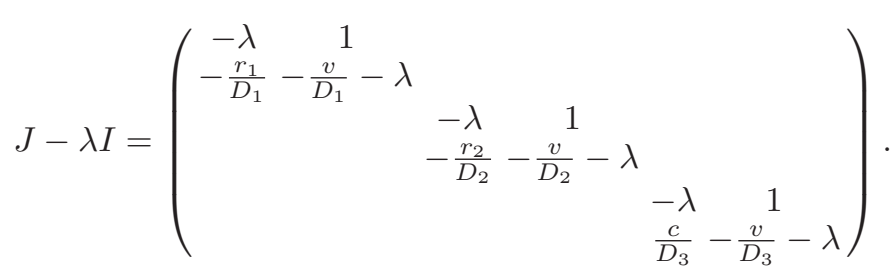

The determinant is thus the product of the determinants of each such block, giving therefore

$$
\left[\lambda\left(\frac{v}{D_{3}}+\lambda\right)-\frac{c}{D_{3}}\right] \Pi_{i=1}^{2}\left[\lambda\left(\frac{v}{D_{i}}+\lambda\right)+\frac{r_{i}}{D_{i}}\right]=0
$$


The eigenvalues are immediately readable, as roots of the quadratic equations arising from each block. For $i=1,2$ we have

$$
D_{i} \lambda^{2}+v \lambda+r_{i}=0,
$$

from which using Descartes' rule of signs, we thus find two negative roots. But from the last one, we have

$$
D_{3} \lambda^{2}+v \lambda-c=0,
$$

which instead has always a positive root. Thus we have shown the following result.

Proposition 4.1. The origin is unstable.

We have further:

Proposition 4.2. $X_{1}$ and $X_{2}$ are also unstable.

Proof. For these cases it is also easy to establish instability.

In fact for $X_{1}$ we have $A_{21}=A_{23}=A_{31}=A_{32}=0$ and $A_{11}=r_{1} D_{1}^{-1}$. With the same procedure we find that the Jacobian is a block upper triangular matrix, from which the eigenvalues again come from the $2 \times 2$ diagonal blocks. In particular the equation

$$
D_{1} \lambda^{2}+v \lambda-r_{1}=0,
$$

by Descartes' rule has one positive eigenvalue.

At $X_{2}$ the Jacobian has the same structure as for $X_{1}$. Instead we have $A_{31}=A_{32}=A_{12}=A_{13}=0$ and $A_{22}=r_{2} D_{2}^{-1}$, so that this time it is the equation

$$
D_{2} \lambda^{2}+v \lambda-r_{2}=0
$$

that has a positive eigenvalue.

Remark 4.3. These results are somewhat expected, since they show that perturbing a system with no populations or only one by adding another one, the system will thrive.

Consider now $X_{3}$.

Proposition 4.4. $X_{3}$ is an unstable equilibrium point.

Proof. Since $A_{12}=A_{13}=0$, here the Jacobian factorizes and the characteristic equation is the product of a quadratic and a quartic. The former,

$$
D_{1} \lambda^{2}+v \lambda-D_{1} A_{11}=0
$$

has roots of uncertain sign, while the latter is

$$
\lambda^{4}+a_{3} \lambda^{3}+a_{2} \lambda^{2}+a_{1} \lambda+a_{0}=0 .
$$

The coefficients $a_{k}, k=0, \ldots 4$ are known. We provide here the most important ones: $a_{0}=A_{22} A_{33}-$ $A_{23} A_{32}$ and

$$
a_{3}=\frac{v}{D_{2} D_{3}}\left(D_{2}+D_{3}\right)>0, \quad a_{1}=-\frac{v}{D_{2} D_{3}}\left(A_{22}+A_{33}\right)=-\frac{c r_{2} v}{D_{2} D_{3} K \delta}<0 .
$$

In view of Descartes' rule, we have at least one negative and one positive root; the latter thus provides instability.

Proposition 4.5. $X_{4}$ is also unstable. 
Proof. We find $A_{21}=A_{23}=0$, so that from the central block of the partitioned Jacobian a quadratic characteristic equation arises, with roots of uncertain sign

$$
D_{2} \lambda^{2}+v \lambda-D_{2} A_{22}=0
$$

The remaining quartic is again (4.6). Its most important coefficients are $a_{0}=A_{11} A_{33}-A_{13} A_{31}$ and

$$
a_{3}=\frac{v}{D_{1} D_{3}}\left(D_{1}+D_{3}\right)>0, \quad a_{1}=-\frac{v}{D_{1} D_{3}}\left(A_{11}+A_{33}\right)=-\frac{c r_{1} v}{D_{1} D_{3} K \beta}<0 .
$$

In view of Descartes' rule, we again find at least one negative and one positive root; the latter thus provides instability.

Proposition 4.6. $X_{5}$ is an unstable equilibrium.

Proof. Note that $A_{31}=A_{32}=0$, so that again the Jacobian factorizes. The quadratic characteristic equation is now

$$
D_{3} \lambda^{2}+v \lambda-D_{3} A_{33}=0 .
$$

but the signs of the roots are not clear. The quartic originating from the remaining part of the Jacobian is once more (4.6). Again, we provide the coefficients whose signs are known, $a_{0}=A_{11} A_{22}-A_{12} A_{21}$,

$$
a_{3}=\frac{v}{D_{1} D_{2}}\left(D_{1}+D_{2}\right)>0, \quad a_{1}=-\frac{v}{D_{1} D_{2}}\left(A_{11}+A_{22}\right) .
$$

Here, observe that the feasibility conditions (4.3) imply that $A_{11}>0$ and $A_{22}>0$, because we have

$$
A_{11}=\frac{r_{1}}{D_{1} \Delta}\left(1-\psi_{1}\right), \quad A_{22}=\frac{r_{2}}{D_{2} \Delta}\left(1-\psi_{2}\right) .
$$

Thus $a_{1}<0$ and by Descartes' rule, there is at least one negative and one positive root, for which instability follows.

Thus, all the boundary equilibrium points in the six-dimensional phase space are unstable, so that any trajectory leaving near them has to remain away from the coordinate hyperplanes.

To complete our analysis, we need to show that the trajectories emanating from these unstable equilibria remain in the feasible region.

Proposition 4.7. The trajectories originating from the unstable boundary equilibria enter into the feasible orthant $\left\{\left(P_{1}, P_{2}, P_{3}\right): P_{i} \geq 0, i=1,2,3\right\}$.

Proof. Let us consider first the origin. Let $\lambda^{*}>0$ be the positive eigenvalue, which originates from the lower right block of the Jacobian. Evidently, $\left(J-\lambda^{*} I\right) \mathbf{u}=\mathbf{0}$ will give a nontrivial solution with $u_{i}=0$, for $i=1, \ldots 4$, because $\lambda^{*}$ is not an eigenvalue of the other two diagonal blocks. In the bottom right block of $J-\lambda^{*} I$, however, the two rows are linearly dependent. We delete the second one, and are left with the equation $-\lambda^{*} u_{5}+u_{6}=0$, which in terms of our original variables is $-\lambda^{*} P_{3}+Q_{3}=0$, and finally, recalling the definition of $Q_{3}$, it becomes the differential equation $P_{3}^{\prime}=\lambda^{*} P_{3}$. Its solution is therefore $P_{3}(s)=P_{3}(0) \exp \left(\lambda^{*} s\right)$. Thus any trajectory leaving from a point near the origin has to lie away from the coordinate hyperplanes, because of the instability of the equilibrium, but it also must remain within the feasible region because biologically we must take the initial condition in this domain, $P_{3}(0)>0$.

Because of the upper triangular block structure of the Jacobian, for the equilibria $X_{1}$ and $X_{2}$ the proof follows the same steps, in turn giving two differential equations whose solutions respectively are $P_{1}(s)=P_{1}(0) \exp \left(\lambda^{*} s\right)$ and $P_{2}(s)=P_{2}(0) \exp \left(\lambda^{*} s\right)$.

At the equilibria $X_{3}, X_{4}$ and $X_{5}$, the positive eigenvalue $\lambda^{*}>0$ is a root of a quartic equation stemming therefore from a suitable $4 \times 4$ submatrix $\widetilde{J}$ of $J$. To be specific, let us take the case of $X_{5}$. 
The submatrix is the top right $4 \times 4$ block of $J$. If $\lambda^{*}$ is an eigenvalue coming from this submatrix, then $P_{3}=0$.

As remarked earlier in Proposition 4.6, the eigenvalues arising from the bottom right block could be of opposite sign, or both negative. In the former case, as argued above, let us take $\lambda_{+}>0$. A sufficient condition for this to occur would be

$$
\left[c\left(1-\psi_{1} \psi_{2}\right)-\beta K\left(1-\psi_{1}\right)-\delta K\left(1-\psi_{2}\right)\right] \Delta>0 .
$$

We have the equation $P_{3}^{\prime}=\lambda_{+} P_{3}$, whose solution is always positive, $P_{3}(s)=P_{3}(0) \exp \left(\lambda_{+} s\right)$, so that the trajectory coming out of the equilibrium remains in the first orthant. If both eigenvalues are negative, $\lambda_{ \pm}<0$, the same equation shows that $P_{3}$ approaches zero with positive values. But the plane $P_{1}-P_{2}$ cannot be crossed because of the existence and uniqueness theorem for ordinary differential equations. The trajectory approaching $X_{5}$ will then move away from it along a direction parallel to the $P_{1}-P_{2}$ plane, given by the eigenvector relative to the positive eigenvalue $\lambda^{*}$ of the quartic characteristic equation of the submatrix $\widetilde{J}$. Thus, the trajectory coming out of the steady state remains in the feasible region.

The cases for the other two equilibria $X_{3}$ and $X_{4}$ are treated in a similar way. Note that sufficient conditions for having $\lambda_{+}>0$ in these cases are respectively $\psi_{1} c \geq \delta K$, for $X_{3}$ and $\psi_{1} c \geq \beta K$, for $X_{4}$. These combined with the feasibility conditions (4.1) and (4.2) gives possible ranges for the zooplankton mortality to ensure positivity of one eigenvalue

$$
\frac{\delta K}{\psi_{1}}<c<\delta K ; \quad \frac{\beta K}{\psi_{2}}<c<\beta K .
$$

Therefore we have demonstrated that the necessary conditions of existence of travelling waves are satisfied. They would originate by perturbing anyone of these boundary equilibrium points and progress toward points in the phase space in which all the populations have nonzero values. In particular, they would reach the interior coexistence equilibrium, when the latter is stable.

\section{Waves for the toxic model}

Now we examine (3.6). In this case the equilibrium $X_{3}=\left(0, P_{2}, P_{3}\right)$ is never feasible, contrary to the former case, while all the other ones present in (3.5) arise here too. As for the other equilibria, we find again the very same points $X_{0}, X_{1}, X_{2}, X_{4}$ and $X_{5}$. The coexistence point instead is different, in fact a double equilibrium stemming from the roots of a quadratic equation, see [2] for more details.

The Jacobian (4.4) changes only in some elements of the last row of $A$, which now becomes

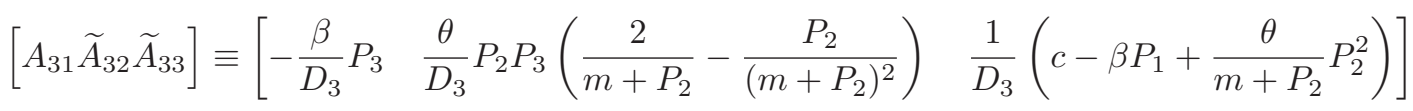

The main result of this Section parallels the findings of the former one.

Proposition 5.1. All the equilibria lying on the boundary of the six dimensional phase space are unstable.

Proof. The origin is unstable, we get the same Jacobian as in the former case.

For $X_{1}$, we find $A_{31}=0, \widetilde{A}_{32}=A_{32}=0, \widetilde{A}_{33}=A_{33}$, so that the eigenvalue analysis remains the same.

At $X_{2}$ the positive eigenvalue comes from the equation (4.5) which is unchanged, and therefore the instability result still holds.

At $X_{4}$ we find $\widetilde{A}_{32}=0, \widetilde{A}_{33}=A_{33}=0$, and the relevant coefficients of the characteristic equation (4.6), in this case denoted by $\widetilde{a}_{i}$, coincide with the ones for the non toxic model,

$$
\widetilde{a}_{1}=a_{1}=-\frac{c r_{1} v}{D_{1} D_{3} K \beta}<0, \quad \widetilde{a}_{3}=a_{3}=\frac{v}{D_{1} D_{3}}\left(D_{1}+D_{3}\right)>0,
$$


so that again we have at least one positive and one negative eigenvalue.

For $X_{5}$ again $\widetilde{A}_{32}=0$ but $\widetilde{A}_{33} \neq A_{33}$. In the quartic (4.6) there is no change in the two relevant coefficients, namely $\widetilde{a}_{1}=a_{1}$ and $\widetilde{a}_{3}=a_{3}$. Hence the existence of at least one positive and one negative eigenvalue is ensured in this case as well.

Again, we have shown that all the boundary equilibria in the phase space are unstable, so that the trajectories must remain away from them. We address now the issue that the trajectories must remain in the feasible region.

Proposition 5.2. The trajectories originating from the unstable boundary equilibria enter into the positive orthant.

We omit the proof of this result, as it parallels the one of the nontoxic case.

\section{Numerical results}

In this section we confirm numerically the emergence of the travelling wave solutions studied earlier and investigate further situations that were not covered by the previous theoretical study.

Equations (2.2) are solved by means of a numerical scheme that employs a spatial discretization based on piecewise-linear continuous finite elements and a fully implicit timestepping scheme (Crank-Nicolson). Mass lumping is employed in order to avoid spurious oscillations arising from the reaction terms.

The parameters in the model are set to the same values used in the one dimensional simulations of [2], namely: $\psi_{1}=0.6, r_{1}=4.0, \alpha_{1}=0.6, \psi_{2}=0.8, r_{2}=5.0, \alpha_{2}=0.7, K=56.0$, for the phytoplanktons and $\beta=0.4, \delta=0.08, c=3.0$, for the zooplankton in the non-toxic case and $\theta=0.2, m=5.0$ in the toxic model. For both the non-toxic and the toxic model, simulations are generally performed in the $[0,100]$ spatial domain. The diffusion coefficients of the zooplankton is set as in [2] to $D_{3}=0.1$. The diffusion coefficients for both phytoplankton species are varied in order to investigate the dependence of the behaviour described here on the relative mobility of the species.

In order to confirm the existence of travelling waves, in each simulation we choose an unstable equilibrium $E_{\mathrm{r}}$ and set the initial value to be $\left.\mathbf{p}(x)\right|_{t=0}=E_{\mathrm{r}}+\epsilon(x)$ where $\epsilon(x)$ is a local perturbation whose role is to throw the system off equilibrium in a small neighborhood of the left domain boundary. The perturbation $\epsilon(x)$ was always chosen to be nonzero only for one species and in particular for one of the species which is absent in $E_{\mathrm{r}}$. In this way, all simulations presented in this section have the ecological interpretation of "invasion" by one species of an ecosystem where one or two other species already coexist in a spatially uniform equilibrium. If the solution is a travelling wave with $\mathbf{p}(-\infty)=E_{\ell}$ and $\mathbf{p}(+\infty)=E_{\mathrm{r}}$, for an equilibrium $E_{\ell} \neq E_{\mathrm{r}}$, the invasion was successfull and the system moves towards the equilibrium $E_{\ell}$ which includes a nonzero density of the new population. If, moreover, the solution of (2.2) with components equal to $E_{\ell}$ is spatially homogeneous, at the left of the travelling wave we will observe a "flat" solution corresponding to the new equilibrium of the ecosystem that includes the newly arrived population.

\subsection{Existence of travelling waves}

In the first set of tests we fixed $D_{1}=1.0$ and $D_{2}=0.5$ as in [2] in order to investigate the existence of travelling waves between different pairs of equilibria. We start from an empty environment (equilibrium $\left.E_{0}\right)$ and show that a travelling wave linking $E_{0}$ to $E_{1}$ exist by setting the initial value to

$$
p_{1}(x)=e^{-x^{2}} \quad p_{2}(x)=0.0 \quad p_{3}(x)=0.0 \quad \text { for } x \in[0,100] .
$$

The top-left panel of Figure 2 shows a series of snapshots of the solution at increasing times. Of course only the first population is present and it tends to invade the whole domain, leaving behind the equilibrium $E_{1}=(56.0,0.0,0.0)$. Similarly the top-right panel of Figure 2 shows the travelling wave solution corresponding to the invasion by a second phytoplankton species $p_{2}$ of an environment where $p_{1}$ is at the 

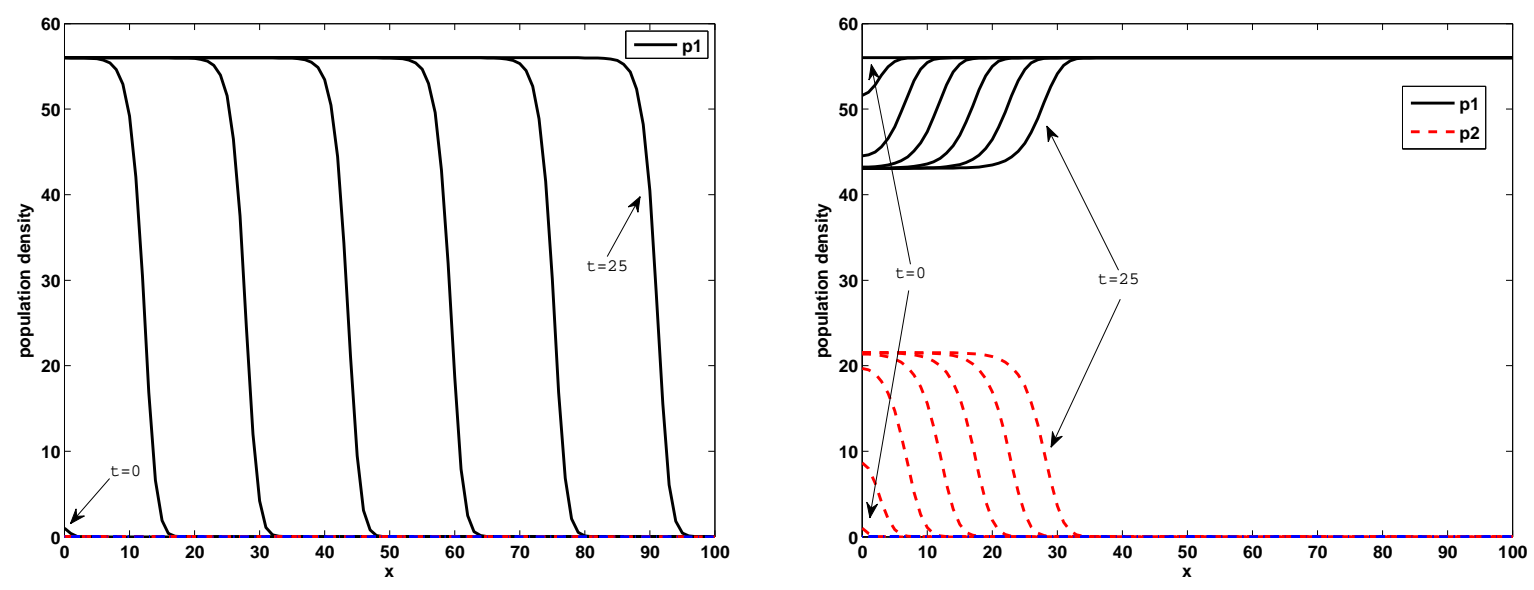

$0 \leq x \leq 100, E_{0} \rightarrow E_{1}$
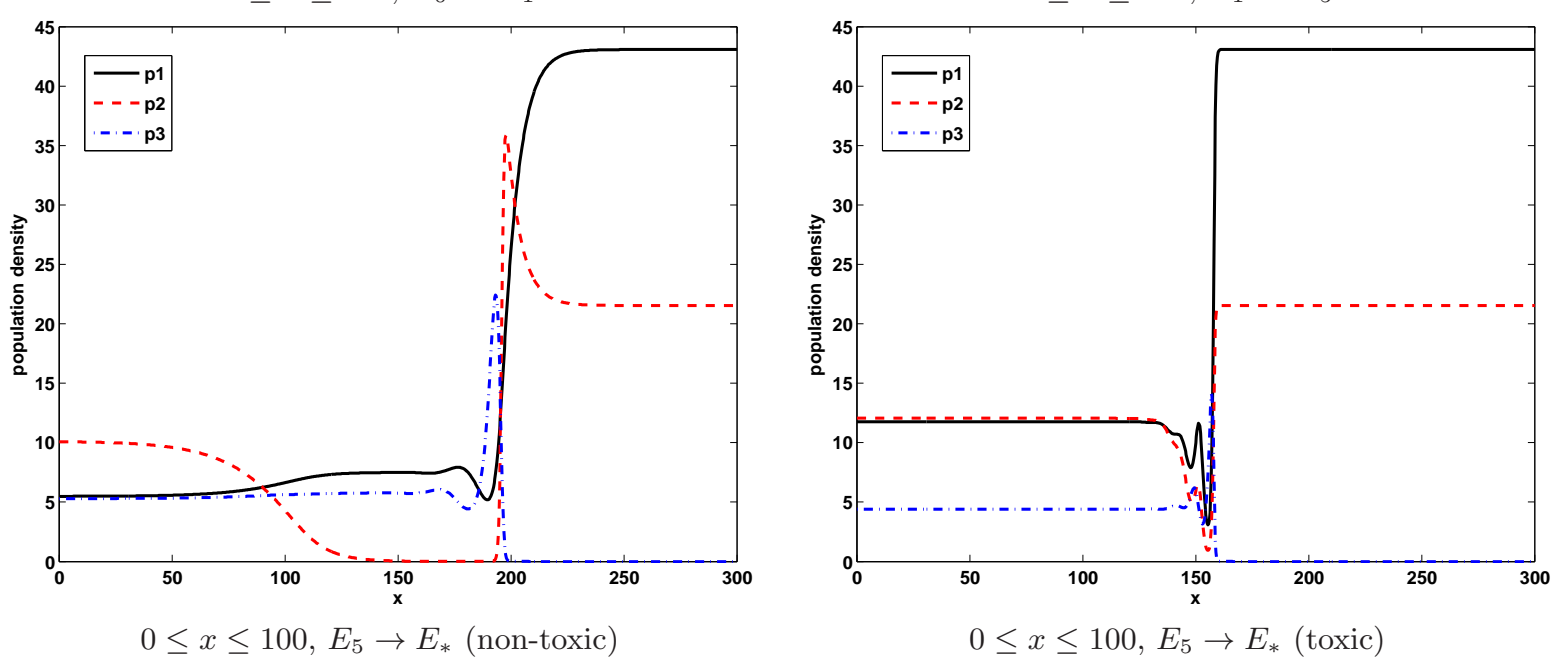

FiguRE 2. Travelling waves between spatially uniform stable equilibria $\left(D_{1}=1.0, D_{2}=\right.$ $0.5, D_{3}=0.1$ ). Top: initial value and six snapshots up to $t=25$ for the invasion of $p_{1}$ into an empty environment (right frame) and for the invasion of $p_{2}$ into an environment where $p_{1}$ is in equilibrium (left frame). Bottom: snapshot at $t=50$ for the invasion of $p_{3}$ into an environment where $p_{1}$ and $p_{2}$ coexist in equilibrium.

spatially uniform equilibrium $E_{1}$. We remark that the speed of this wave is much slower than the previous one. Finally, these two kinds of waves are identical in both the toxic and in the non-toxic models, the third population $p_{3}$ is in fact absent and thus no difference arise between the models.

The lower panels of Figure 2 show the transitions between $E_{5}$ and $E_{*}$. The initial data are

$$
p_{1}(x)=43.08 \quad p_{2}(x)=21.54 \quad p_{3}(x)=e^{-x^{2}} \quad \text { for } x \in[0,100]
$$

In this case, the interaction between $p_{2}$ and $p_{3}$ plays a role and thus the difference between the toxic and non-toxic model is apparent. However, for $D_{1}=1$, the spatially homogeneous internal equilibrium $E_{*}$ is stable also for the toxic model and thus the travelling wave leaves behind a spatially homogeneous state, namely $(5.47,10.14,5.29)$ for the non-toxic model (bottom left in the figure) and $(11.75,12.05,4.41)$ for the toxic model (bottom right in the figure). The snapshots shown are taken at $t=50$ and the computational 
domain has been enlarged to $[0,300]$ in order to show completely the whole transition, which has a much more complex shape than the previous ones.

\subsection{The role of the diffusion coefficient}

Next we investigate the transition from $E_{4}$ to $E_{*}$ for both models, varying the diffusion coefficients $D_{1}$ and $D_{2}$. Initial data were set as a small perturbation of $E_{4}$, namely:

$$
p_{1}(x)=7.50 \quad p_{2}(x)=e^{-x^{2}} \quad p_{3}(x)=5.77 \quad \text { for } x \in[0,100]
$$

The ecological interpretation of this initial condition is the arrival (from the left boundary at $x=0$ ) of a second phytoplankton species into an ecosystem where one phytoplankton and the zooplankton population already coexist in equilibrium. In all cases we observe the emergence of a travelling wave solution that represents the invasion of the ecosystem by the new phytoplankton population. The solution has a rightmoving front that separates the internal coexistence equilibrium $E_{*}$ (on its left) and the unstable $E_{5}$ equilibrium (on its right). The evolution of the travelling waves is rendered by a sequence of snapshots in Figure 3 for the cases of $D_{1}=1$ (left) and $D_{1}=100$ (right). One can note that the diffusion coefficient does not change the speed of the wave, but it influences the shape of the transition. This is even more evident in Figure 4, where the solutions at $t=60$ are compared for $D_{1}$ ranging from 1 to $10^{3}$. In all four cases the "center" of the transition is located at around $x=50$, while the width of the transition and the presence of oscillations inside it increases remarkably with the increase of the relative mobility of the species $p_{1}$ with respect to the other ones. We also note that changing the value of $D_{2}$ does not have significant effects on the shape of the waves.

In the toxic case, the situation is very different, since the initial datum (6.1) represents the arrival in the ecosystem of a phytoplankton species $p_{2}$ on which the zooplankton $p_{3}$ feeds but which is toxic for $p_{3}$. Depending on the value of the diffusion coefficients $D_{1}$ and $D_{2}$, from [2] it is known when the internal equilibrium is spatially homogeneous. For $D_{2}=0.5$ and $D_{3}=0.1$, the threshold for instability is found at $D_{1}=1.02$, while for $D_{1}=1.0$ and $D_{3}=0.1$, the threshold for instability is found at $D_{2}=0.23$

The left column of Figure 5 shows snapshots of the evolution in the case $D_{1}=1$. In this case, apart from the numerical values of the internal equilibrium, the solution is comparable with the non-toxic case shown in Figure 3. The right column of 5 shows snapshots of the evolution for a value of the diffusion coefficient $\left(D_{1}=100\right)$ that is above the threshold for instability of the internal equilibrium. This situation is not covered by the theory exposed in the previous sections, but nevertheless we observe a sort of travelling wave, except that at the left of the transition the non spatially uniform equilibrium already observed in [2] emerges.

Figure 6 shows the solutions at $t=60$ for different values of the diffusion coefficients. For $D_{1}=1$ we observe the appearence of the (stable) spatially uniform internal equilibrium, while for the other cases the solution at the left of the transition is stationary but not spatially uniform. The population densities present oscillations that alternate areas of high concentrations of the toxic species $p_{2}$ with high concentrations of the zooplankton $p_{3}$. In areas where $p_{3}$ is less present, also $p_{1}$ has higher concentrations, due to reduced feeding by $p_{3}$. Increasing $D_{1}$, the wavelength of the spatial oscillations increases. It is remarkable that the mobility of $p_{1}$, on its own, can influence the wavelength of the oscillations between the populations $p_{2}$ and $p_{3}$. The two lower panels of Figure 6 present the case where $D_{1}$ is kept at the value used in [2], but $D_{2}$ is lowered below the instability threshold. We observe the emergence of another non spatially-uniform equilibrium, with characteristics similar to the ones observed above.

\section{Conclusions}

We investigate spatially-dependent models of the interaction between two phytoplankton species and one zooplankton population that feeds on the previous two. In particular we consider two cases, distinguished by the toxicity of the second phytoplankton species for the zooplankton. A previous paper [2] had already investigated the stability of spatially uniform equilibria, showing that, when one of the 

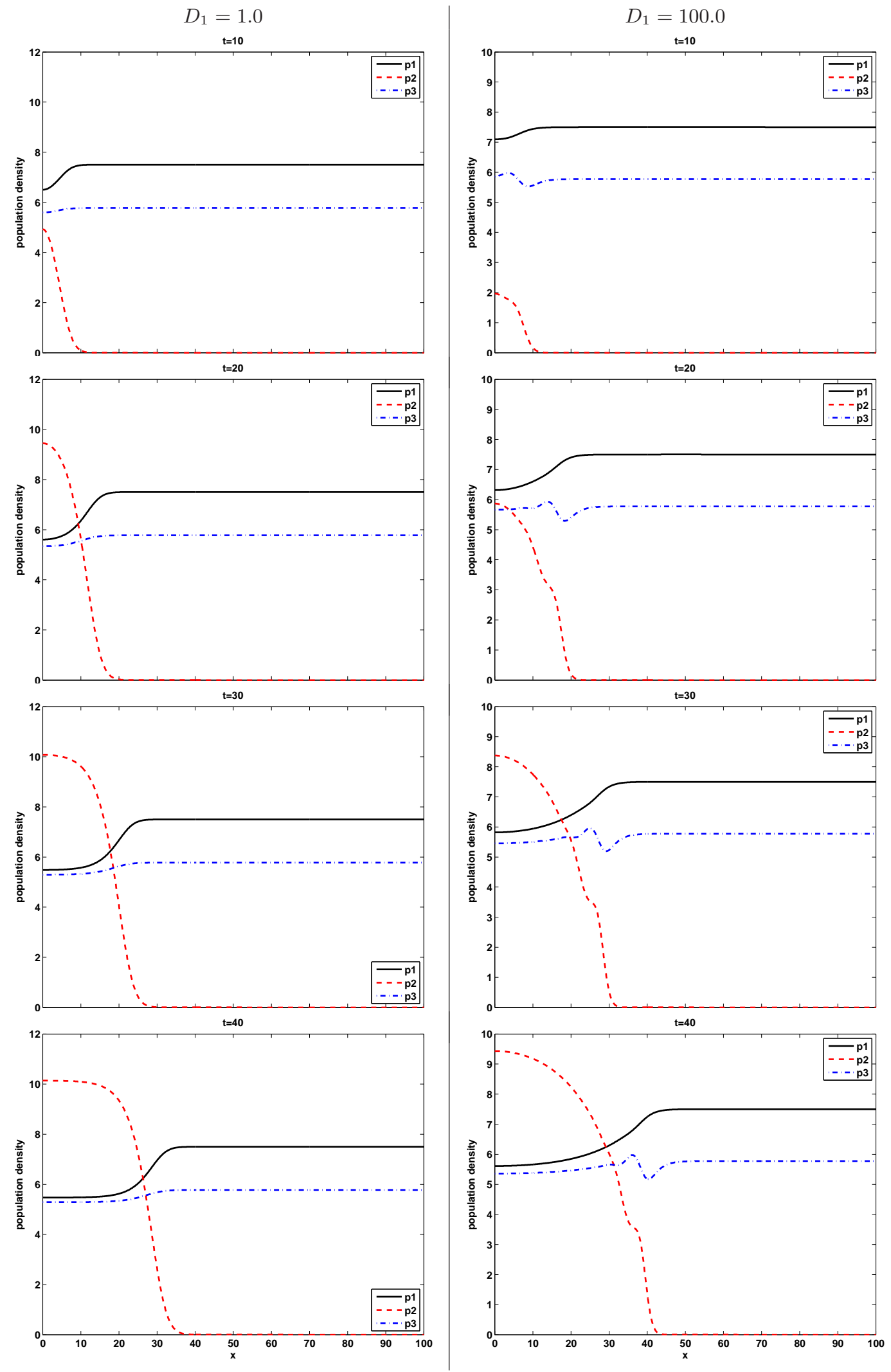

FiguRE 3 . Snapshpots for the travelling wať linking $E_{4}$ and $E_{*}$ for the non-toxic model. 

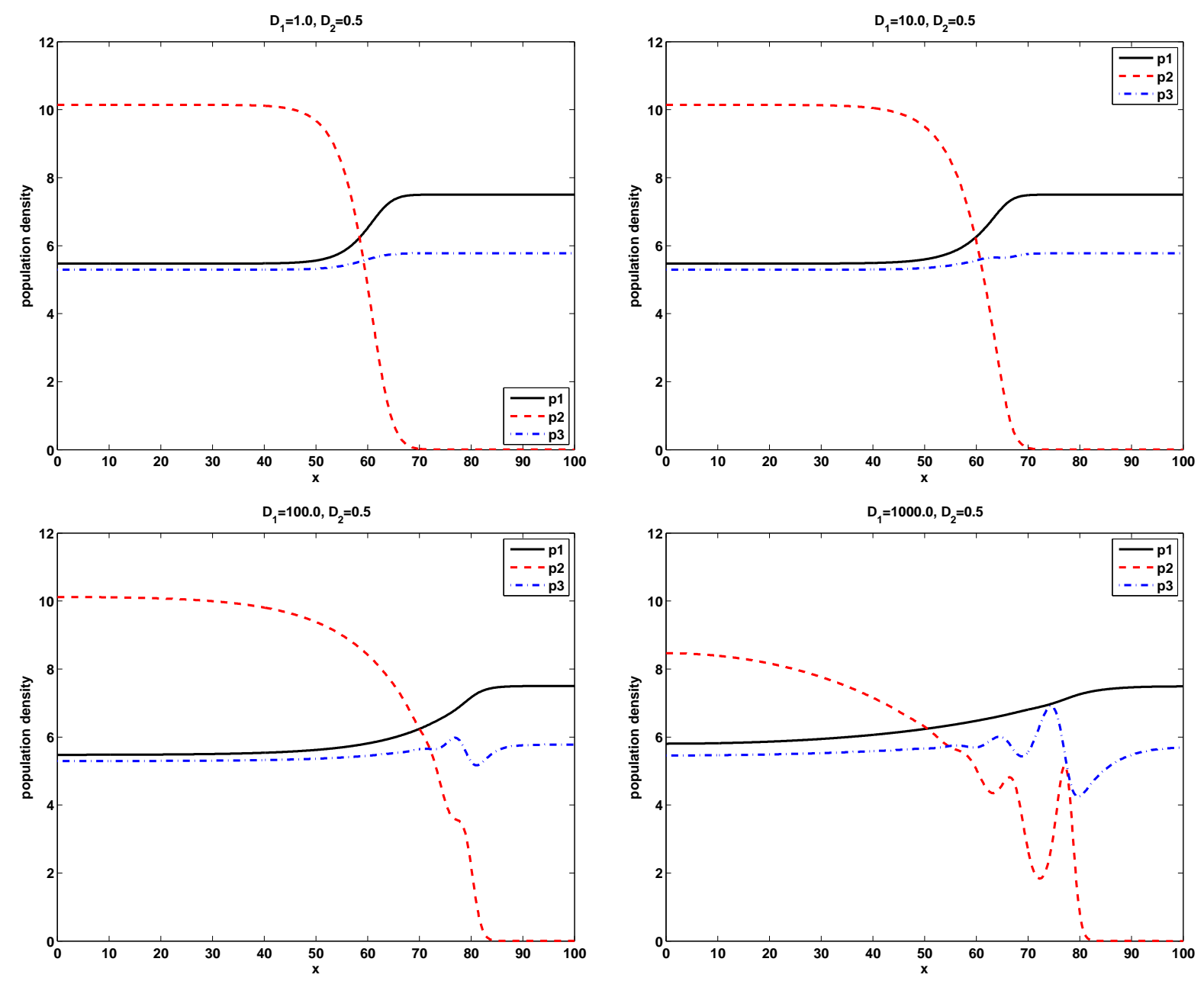

Figure 4 . Snapshpots at $t=60$ for the travelling wave linking $E_{4}$ and $E_{*}$ in the nontoxic model.

phytoplankton species is toxic for the zooplankton, the spatially uniform coexistence equilibrium is stable only for moderate values of the relative mobility of the phytoplankton species. This result was confirmed numerically, showing that random initial conditions could lead to the emergence of non spatially-uniform stable equilibria and this result was related to the ecological phenomenon of red tides.

In this paper we consider the more realistic situation in which the system is in equilibrium when a new species appears at a single location in the domain, either due to a point contamination of the environment or coming from a nearby ecosystem. Mathematically, this perturbation throws the system off equilibrium at a single location in space (e.g. on the boundary) and may give rise to an invasion wave. The existence of such travelling waves are investigated both analytically and numerically. The analytical study shows the existence of travelling waves linking pairs of equilibria of the system and is limited to the regimes where spatially uniform equilibria exist. The numerical study confirms the previous findings and additionally investigates the other regime too, showing examples of traveling waves that leave behind them the non spatially-uniform coexistence equilibrium of the toxic model. 

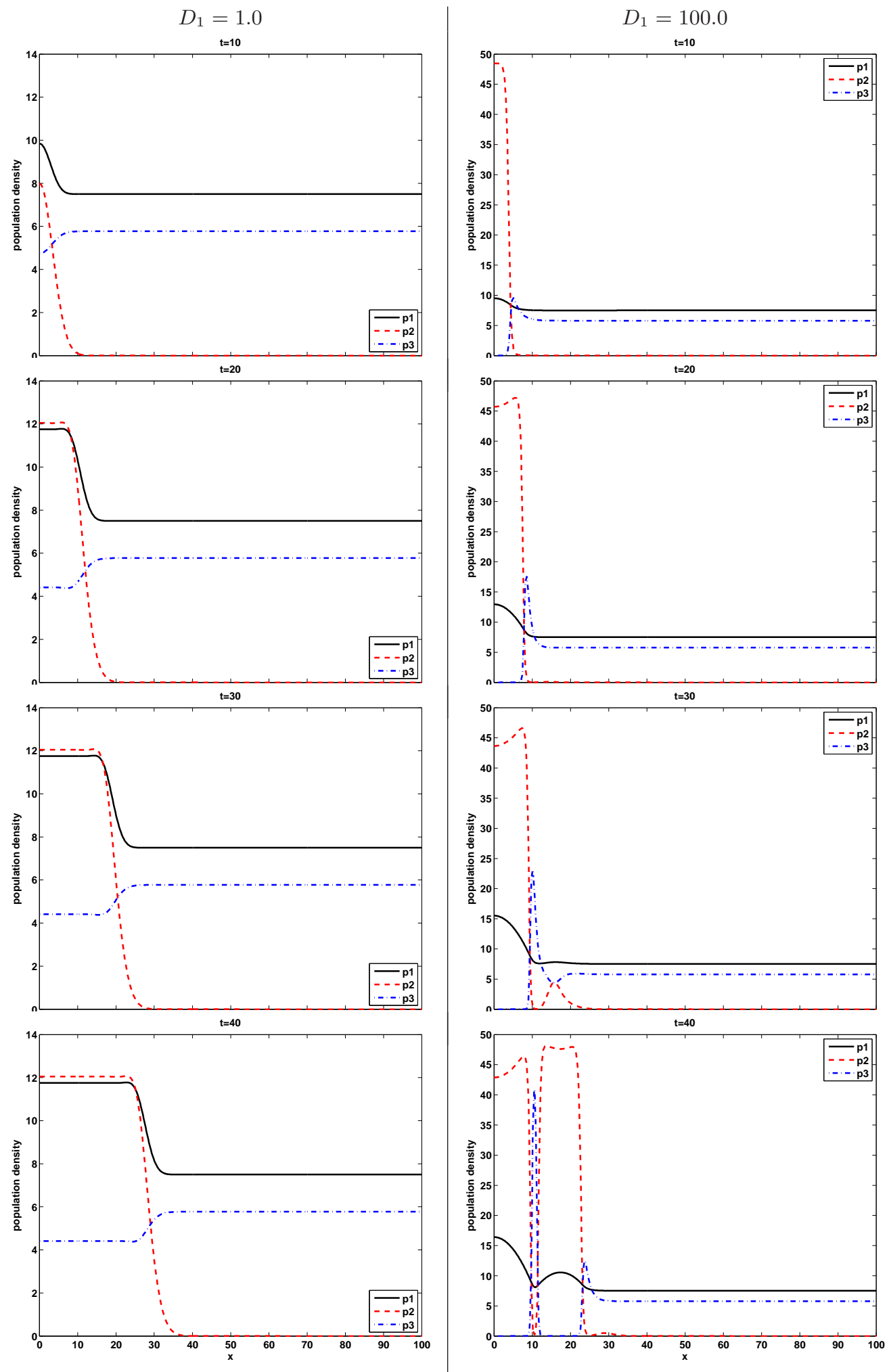

Figure 5. Snapshpots for the travelling wave linking $E_{4}$ and $E_{*}$ for the toxic model for $D_{2}=0.5$. Note the different vertical scales. 

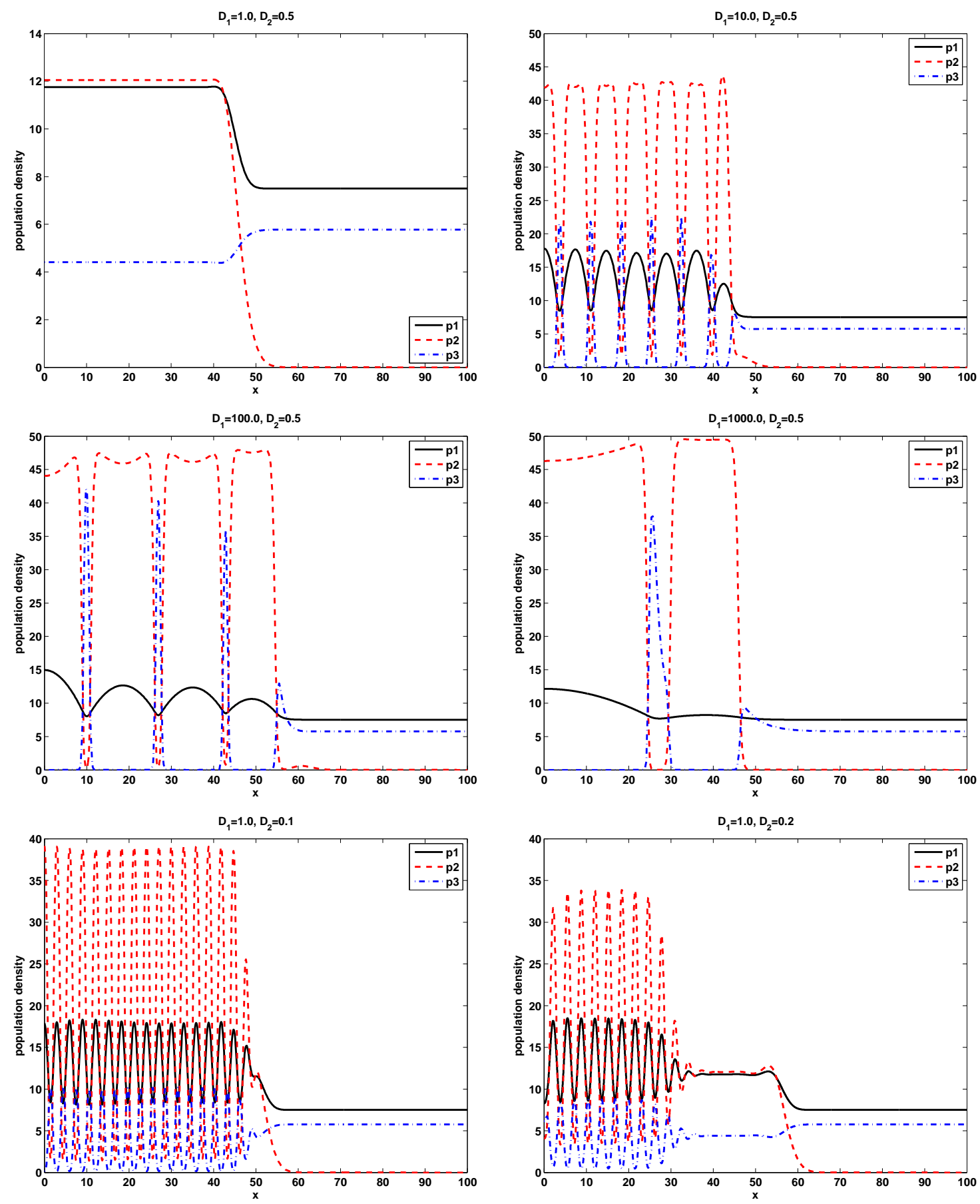

Figure 6 . Snapshpots at $t=60$ for the travelling wave linking $E_{4}$ and $E_{*}$ in the toxic model. In the first four panels, $D_{1}$ was varied, $D_{1}=1$.0, $D_{1}=10, D_{1}=100, D_{1}=1000$; in the lower two, $D_{2}$ is changed, $D_{1}=1.0, D_{2}=0.1$ and $D_{1}=1.0, D_{2}=0.2$. 


\section{References}

[1] D. M. L. Anderson. Toxic algae blooms and red tides: a global perspective. In Red Tides: Biology, Environmental Science and Toxicology, T. Okaichi, D. M. Anderson, T. Nemoto (Editors). Elsevier: New York, USA (1989), 11-21.

[2] S. Chaudhuri, J. Chattopadhyay, E. Venturino. Toxic phytoplankton-induced spatiotemporal patterns. J. Biol. Phys., 38 (2012), 331-348.

[3] S. Chaudhuri, S. Roy, J. Chattopadhyay. Comparison of plankton dynamics in the 'presence' or 'absence' of toxic phytoplankton. Appl. Math. Comput., sumbitted for publication.

[4] J. Chattopadhyay, R. R. Sarkar, S. Mandal. Toxin-producing plankton may act as a biological control for planktonic blooms- field study and mathematical modelling. J. Theor. Biol., 215, (2002), 333-334.

[5] J. Chattopadhyay, R. R. Sarkar, A. El Abdllaoui. A delay differential equation model on harmful algal blooms in the presence of toxic substances. IMA J. Math. Appl. Med. Biol., 19, (2002), 137-161.

[6] G. M. Hallegraeff. A review of harmful algae blooms and the apparent global increase. Phycologia, 32, (1993), 79-99.

[7] H. Malchow, S. Petrovskii, E. Venturino. Spatiotemporal patterns in Ecology and Epidemiology. CRC, Boca Raton, 2008.

[8] S. Roy, S. Alam, J. Chattopadhyay. Competitive effects of toxin-producing phytoplankton on overall plankton populations in the Bay of Bengal. Bull. Math. Biol., 68(8), (2006), 2303-2320.

[9] S. Roy, S. Bhattacharya, P. Das, J. Chattopadhyay. Interaction among non-toxic phytoplankton, toxic phytoplankton and zooplankton: inferences from field observations. J. Biol. Phys., 33(1) (2007), 1-17.

[10] S. Roy. Spatial interaction among nontoxic phytoplankton, toxic phytoplankton, and zooplankton: emergence in space and time. J. Biol. Phys., 34, (2008), 459-474.

[11] T. Smayda. Novel and nuisance phytoplankton blooms in the sea: evidence for a global epidemic. In Toxic Marine Phytoplankton, E. Graneli, B. Sundstrom, L. Edler, D. M. Anderson (Editors), Elsevier, New York, USA, 1990, $29-40$. 